\title{
Effects of multimodal exercise on clinical status and patient-reported outcomes in children undergoing hematopoietic stem cell transplantation
}

\section{Vesile Yildiz Kabak, Duygu Uckan Cetinkaya, Baris Kuskonmaz, Nevin Cetin \& Tulin Duger}

To cite this article: Vesile Yildiz Kabak, Duygu Uckan Cetinkaya, Baris Kuskonmaz, Nevin Cetin \& Tulin Duger (2019) Effects of multimodal exercise on clinical status and patient-reported outcomes in children undergoing hematopoietic stem cell transplantation, Pediatric Hematology and Oncology, 36:7, 410-421, DOI: 10.1080/08880018.2019.1648619

To link to this article: https://doi.org/10.1080/08880018.2019.1648619

\section{曲 Published online: 18 Sep 2019.}

\section{Submit your article to this journal $\square$}

Џlll Article views: 104

Q View related articles ¿

View Crossmark data ¿ 


\title{
Effects of multimodal exercise on clinical status and patient-reported outcomes in children undergoing hematopoietic stem cell transplantation
}

\author{
Vesile Yildiz Kabak ${ }^{a}$, Duygu Uckan Cetinkaya ${ }^{b}$, Baris Kuskonmaz ${ }^{b}$, Nevin Cetin ${ }^{c}$, \\ and Tulin Duger ${ }^{\mathrm{a}}$ \\ ${ }^{a}$ Faculty of Physical Therapy and Rehabilitation, Hacettepe University, Ankara, Turkey; ${ }^{b}$ Faculty of \\ Medicine, Department of Pediatrics, Division of Bone Marrow Transplantation Unit, Hacettepe \\ University, Ankara, Turkey; ' Ihsan Dogramaci Children Hospital, Hacettepe University, Ankara, Turkey
}

\begin{abstract}
There are limited data available regarding effectiveness of exercise training in children undergoing hematopoietic stem cell transplantation (HSCT). We aimed to investigate effects of multimodal exercise program on clinical status and patient-reported outcomes including pain, fatigue, depression, and quality of life (QOL) level of children and their parents' QOL level. Twenty-six children undergoing HSCT participated in this study. Clinical status, pain, fatigue, depression, and QOL level of children were assessed three times: before HSCT, at discharge, and one month later. For intervention group (IG, $n=15)$, multimodal exercise program was performed five days a week, throughout hospitalization and children were advised to continue exercise program at home. For control group (CG, $n=11)$, being active as much as possible was advised. The number of painful day and pain intensity was significantly lower in IG than in CG during hospitalization $(p<.05)$. Depression level decreased in IG at the time points $(p<.05)$; however, there was no significantly difference between groups. The QOL level was higher in IG than CG only at control measurements $(p<.05)$. In addition, QOL level of the parents decreased in both groups $(p<.05)$. There was no statistically difference between groups in terms of other clinical variables. The multimodal supervised exercise program has positive effects on children's pain and QOL level. Exercise program was also well tolerated by children during hospitalization. In addition, QOL levels of the parents were also negatively affected during hospitalization, and interventions aiming to increase $\mathrm{QOL}$ level of the parents should be considered.
\end{abstract}

\section{ARTICLE HISTORY}

Received 15 February 2019

Revised 16 July 2019

Accepted 20 July 2019

\section{KEYWORDS}

Children; emotional status; exercise; hematopoietic stem cell transplantation; quality of life

\section{Introduction}

Hematopoietic stem cell transplantation (HSCT) has been frequently performed for the treatment of hematopoietic and immune system disorders and some kinds of cancers. ${ }^{1}$ With the improvement of HSCT treatment options, survival rate has increased rapidly in recent years. The survival rate for patients treated with HSCT has reached $80 \%$ in 
some conditions. ${ }^{2}$ As a result, side effects of this treatment and patients' perception of quality of life (QOL) have become more important.

In spite of the improvement in treatment approaches, HSCT is still considered as an aggressive therapy which results with functional, psychological, and social impairments. ${ }^{3-6}$ During the inpatient period, patients suffer from several side effects including pain, fatigue, fever, vomiting, diarrhea, and constipation. Besides these common symptoms, graft-versus-host disease, long hospitalization period, and organ toxicities led to reduce in physical function and increase in fatigue complaint, and as a result decrease in patients' QOL level. ${ }^{7,8}$ In addition, there are various studies reported reduction in psychological status and QOL level when compared with the pretransplantation level in adult and pediatric HSCT patients. ${ }^{9,10}$

With the increasing numbers of survivors, requirement is increasing for the treatment options, which help patients to overcome side effects and to increase their QOL. In a study investigating effects of supervised exercise program during HSCT, positive effects of exercise program were found on patients' global distress, fatigue, anger, and hostility. ${ }^{11}$ According to results of a review regarding this topic, exercise seems as a beneficial intervention to decrease treatment-related complications and to contribute patients' QOL level. ${ }^{9}$

When compared with adult patients, there are fewer publications which investigate effectiveness of exercise training for children undergoing HSCT. ${ }^{12-15}$ In these studies, exercise programs had beneficial effects on physical functions and QOL during and after HSCT. ${ }^{12-14}$ According to a recent review, more evidence is needed to draw more precise results regarding rehabilitation of pediatric HSCT patients. ${ }^{16}$ In our previous study, individual supervised exercise program during hospitalization and after discharge continued with home exercise program had positive effects on physical capacity, functional mobility, and muscle strength of the children undergoing HSCT. ${ }^{15}$ In the present study, we will further examine effects of multimodal exercise program on clinical status, emotional status, and QOL level in pediatric HSCT patients. In addition, we aimed to analyze QOL level of caregivers during HSCT period.

\section{Material and methods}

This prospective study was conducted in Ankara/Turkey. In the present study, a supervised multimodal exercise intervention was performed and results were compared with a control group. Totally, 26 children who underwent HSCT in Hacettepe University Ihsan Doğramacı Children Hospital and Ankara Children Health and Diseases Hematology Oncology Education and Research Hospital were participated. Transplantation process was established by the same clinical director in both hospitals; thus protocols were shared between both transplant units. Inclusion criteria were the following: children between 3 and 17 years of age, who can cooperate and were admitted to the hospital for HSCT. Exclusion criteria were the following: serious impairment of cardiovascular, pulmonary, cognitive, emotional, neurologic, or locomotor systems. Eligible children were divided into two groups as intervention group (IG, $n=15$ ) and control group (CG, $n=11$ ). There were no routine physiotherapy and exercise programs in both transplant units. A structured, individual, and supervised multimodal 
exercise program was conducted for IG, and being active and moving as much as possible was recommended for CG. CG received oral information by the physiotherapist regarding being active. They advised to move and/or walk as much as possible during inpatient period and activities such as walking or cycling were advised after discharge. Children were assessed everyday during inpatient period in terms of clinical status and they were assessed at three different time points: at baseline (the first three days of hospitalization $-t_{1}$ ), at discharge (the last two days of hospitalization $-t_{2}$ ), and one month later from discharge $\left(t_{3}\right)$. Approval of the Ethical Committee of Hacettepe University was obtained. Before entering the assessment, informed consent was signed by each child or their parents.

\section{Outcome variables}

\section{Clinical status}

Children's clinical status was recorded every day during the inpatient period. The hematologic parameters (hemoglobin level, thrombocyte level, neutrophil count) of the patients were analyzed routinely during HSCT period. The presence of anemia, thrombocytopenia, neutropenia, total parenteral nutrition support was recorded everyday from patients' hospital records. The presence of nausea, vomiting, diarrhea, constipation, pain, and fatigue was analyzed by questioning patients and parents (Yes/No) and the total number of days which patients suffering from these symptoms was noted.

\section{Depression}

To assess depressive symptoms of the children, the Children's Depression Inventory was used. This inventory consists of 27 items, self-rated, and symptom-oriented. Each item is scored between 0 and 2 points. The total score is between 0 and 54 points and higher scores indicate higher depression level. The Turkish reliability and validity of this inventory were performed. ${ }^{17,18}$

Pain and fatigue. The Wong-Baker FACES Pain Rating Scale was used to assess children's pain and fatigue level. This scale is scored between 0 and 10 points. With this scale, children were asked to point out their pain and fatigue by showing visual expressions. ${ }^{19}$ Intensity of pain and fatigue was assessed daily during inpatient period and these symptoms were also evaluated to detect differences between groups at baseline, at discharge, and one month later from discharge.

Quality of life level of the children. The Pediatric Quality of Life Inventory (PedsQL) Cancer Module was used to assess QOL level of the children. ${ }^{20}$ This inventory has two report forms. One is for children to report their self-QOL and another one is administered to the parents and asks parents to report their children's QOL level. The PedsQL Cancer Module includes 27 items, which is scored between 0 and 100 points, and higher scores indicate better QOL. PedsQL Cancer Module includes different modules according to age groups: 2-4 years, 5-7 years, 8-12 years, and 13-18 years. The Turkish reliability and validity of the PedsQL Cancer Module were performed. ${ }^{21,22}$ 
Quality of life level of the parents. The Nottingham Health Profile was used to assess QOL level of the caregivers. This questionnaire includes 38 items, which are answered as "Yes" or "No." 23 The Nottingham Health Profile is scored between 0 and 100 points and lower scores represent higher QOL. The Turkish validation of this questionnaire was performed by Kucukdeveci et al. ${ }^{24}$

\section{Exercise program}

The individual and structured exercise program including strengthening, endurance, stretching, and relaxation exercises was performed by the same physiotherapist during every weekday throughout hospitalization. According to the hematologic status, clinical status, and vital signs of the children, exercise program was modified day by day. In some days, children were not allowed to do exercise. The reasons that children were not allowed to do exercise were severe thrombocytopenia $\left(<20.000 \mathrm{~mm}^{3}\right)$ severe anemia $(<7 \mathrm{gr} / \mathrm{dl})$, fever $\left(>38.5^{\circ} \mathrm{C}\right)$, severe nausea, and vomiting. We used the Borg rating of perceived exertion scale for estimating the intensity of the exercise program. Exercise intensity was light to moderate intensity according to the Borg rating of perceived exertion (10-13 points). The exercise program was taught to children and their parents and they were advised to continue exercises in weekends. At discharge, home exercise program was generated by physiotherapist and children and their parents were advised to continue exercise program at home.

Strengthening exercises for upper extremity (shoulder flexion-abduction-adductionextension, elbow flexion-extension), lower extremity (hip flexion-abduction-adductionextension, knee flexion-extension, dorsi- and plantar flexion), and trunk (trunk flexion and extension) were performed. Children's own weights were generally used during strengthening exercises. Yellow or red Therabands were used for children who can tolerate. Each exercise was performed as one or two sets with 10-15 repetitions. Endurance exercises were consisted of brisk walking and go up and down on a step board. Brisk walking was conducted inside the hospital room for 5-10 minutes. Step up and down exercise was implemented as 15-20 times in 2-3 sets and if necessary breaks were given between the sets. In order to make exercises more fun, the games with ball and jumping activities were implemented. Stretching exercises for gross muscle groups were implemented 1-2 sets and 10-15 repetitions. The exercises were performed at the prolonged position of the muscles, waiting for 10-15 seconds, and totally 10-15 minutes. In addition to these exercises, relaxation methods and exercises were performed 1-2 times a week for 10-15 minutes by physiotherapist. These exercises were consisted of breathing control, relaxation positions, and relaxation exercises. The difference between contraction and relaxation was taught to children during these methods and relaxation status was tried to be provided.

\section{Data analysis}

Statistical Package for Social Sciences 15.0 software (SPSS Inc., Chicago, IL) was used for statistical analyses. The number of participants was determined by calculation of power analysis with $80 \%$ power with 0.05 level of significance and planned to include at least eleven children for each group, respectively. The Kolmogorov-Smirnov test was used to determine normality of distribution. In order to evaluate the effect of time in 
Table 1. Clinical status of the children during hospitalization.

\begin{tabular}{|c|c|c|c|c|c|}
\hline \multirow[b]{2}{*}{ Outcomes, number of days } & \multicolumn{2}{|c|}{$I G(n=15)$} & \multicolumn{2}{|c|}{$C G(n=11)$} & \multirow[b]{2}{*}{$p$ value } \\
\hline & M & $S D$ & M & $S D$ & \\
\hline Hospitalization, days & 38.92 & 8.82 & 41.16 & 10.20 & 0.765 \\
\hline Anemia, days & 28.75 & 9.96 & 28.85 & 14.91 & 0.482 \\
\hline Thrombocytopenia, days & 26.58 & 9.64 & 28.14 & 15.79 & 0.902 \\
\hline Neutropenia, days & 23.41 & 9.5 & 24.00 & 17.15 & 0.592 \\
\hline TPN, days & 3.20 & 2.25 & 5.50 & 1.37 & 0.073 \\
\hline Nausea, days & 6.46 & 4.44 & 7.40 & 7.01 & 0.976 \\
\hline Vomiting, days & 4.15 & 2.93 & 5.70 & 2.86 & 0.208 \\
\hline Diarrhea, days & 2.61 & 2.29 & 4.20 & 3.82 & 0.605 \\
\hline Constipation, days & 2.15 & 2.93 & 0.90 & 2.02 & 0.284 \\
\hline Painful, days & 2.53 & 3.55 & 8.22 & 5.84 & $0.021^{*}$ \\
\hline Pain level $(0-10)$ & 1.19 & 1.77 & 3.47 & 2.04 & $0.025^{*}$ \\
\hline Fatigue, days & 11.23 & 9.77 & 11.77 & 10.59 & 0.794 \\
\hline Fatigue level (0-10) & 5.59 & 5.51 & 4.31 & 2.06 & 0.140 \\
\hline
\end{tabular}

TPN: Total Parenteral Nutrition, IG: Intervention Group, CG: Control Group, M: Mean, SD: Standard Deviation. MannWhitney U test.

$*_{p}<.05$.

both groups, Friedman test was used and to compare groups at the time points, Mann-Whitney $U$-test was used. The Bonferroni correction was performed to decrease Type I error for post hoc analysis. Wilcoxon test was used to compare before HSCT and at the discharge measurements of the QOL level of caregivers. The level of significance was 0.05 for all statistical analyses and results were expressed as $\%$ or mean $\pm S D$.

\section{Results}

Twenty-six children (IG:15, CG:11) whose mean age was $9.5 \pm 3.08$ years in IG and $6.72 \pm 2.96$ years in CG were included in the present study $(p>.05)$. Baseline body mass index (IG: $16.87 \pm 2.51 / \mathrm{CG}: 15.81 \pm 2.50$ ) was similar between the groups. Children were diagnosed with thalassemia major $(n=5)$, leukemia $(n=4)$, aplastic anemia $(n=2)$, immune deficiency $(n=2)$, and other $(n=2)$ in IG; and leukemia $(n=4)$, aplastic anemia $(n=3)$, and other $(n=4)$ in CG. The transplantation types of the children were allogeneic (IG: 15, CG: 8) and autologous (CG: 3). The preparative regiments of HSCT were myeloablative (IG: 11, CG: 8) and reduced intensity (IG: 4, CG: 3). Busulfan and cyclophosphamide \pm melphalan were used in myeloablative regimens; fludarabine, cyclophosphamide, and antithymocyte globulin were used in reduced intensity regimens. After HSCT, graft-versus-host disease was occurred in one child in IG and in two children in CG. Total score of the depression, pain, fatigue, and QOL was similar in both groups at the baseline. The mean participation rate to the exercise sessions was $72.86 \%$ in IG. During $t_{3}$ measurements, children and/or their parents were verbally asked whether children continue exercise program at home. Only two children in IG reported to regularly continue exercise program at home.

Clinical status of the children during hospitalization period (mean 39 days) is presented in Table 1. During hospitalization period, only the mean number of painful day and the mean pain level were statistically higher in CG than in IG $(p<.05)$. Groups were similar in terms of other variables of clinical status during hospitalization period.

Depression, pain, and fatigue level of the children before HSCT, at the discharge and one month later, are shown in Table 2. Depression level decreased in IG, and according 
Table 2. Depression, pain and fatigue level of the children at baseline, at discharge and one month later.

\begin{tabular}{|c|c|c|c|c|c|c|c|c|}
\hline \multirow[b]{2}{*}{ Outcomes } & \multirow[b]{2}{*}{ Groups } & \multicolumn{2}{|c|}{ Baseline } & \multicolumn{2}{|c|}{ At the discharge } & \multicolumn{2}{|c|}{ One month later } & \multirow[b]{2}{*}{$p$ value } \\
\hline & & M & $S D$ & M & $S D$ & M & $S D$ & \\
\hline \multirow{3}{*}{ CDI Score } & IG & 6.25 & 2.63 & 4.63 & 2.33 & 3.87 & 3.13 & $0.009 *$ \\
\hline & CG & 11.20 & 6.41 & 9.00 & 2.91 & 6.80 & 3.96 & 0.074 \\
\hline & $\mathrm{p}$ & 0.771 & & 0.085 & & 0.222 & & \\
\hline \multirow[t]{3}{*}{ Pain level $(0-10)$} & IG & 0.71 & 1.48 & 0.00 & 0.00 & 0.00 & 0.00 & 0.135 \\
\hline & CG & 0.00 & 0.00 & 0.22 & 0.66 & 0.00 & 0.00 & 0.368 \\
\hline & $p$ & 0.373 & & 0.748 & & 1.00 & & \\
\hline \multirow[t]{3}{*}{ Fatigue level $(0-10)$} & IG & 1.21 & 2.15 & 0.20 & 0.63 & 0.00 & 0.00 & 0.223 \\
\hline & CG & 1.78 & 2.90 & 1.33 & 2.00 & 1.77 & 1.85 & 0.584 \\
\hline & $p$ & 0.900 & & 0.468 & & 0.059 & & \\
\hline
\end{tabular}

CDI: Children's Depression Inventory, IG: Intervention group, CG. Control group, M: Mean, SD: Standard Deviation. Friedman test, Mann-Whitney U test.

$*_{p}<.05$.

to the post hoc tests, this was statistically significant between at baseline and at control measurements $\left(t_{1}-t_{3}, p=.011\right)$. However, according to the group comparisons, IG and CG were similar in terms of depression level. According to pain assessments, there was no statistically significant difference between groups at all time points (Table 2). When we look at the fatigue level of the children, there was slightly significant difference at $t_{3}$ and IG had lower fatigue level than CG. On the other hand, there was low level of fatigue in both groups at all time points (Table 2).

The QOL level of the children at baseline, at the discharge, and one month later is shown in Table 3. In IG, according to the PedsQL-Child Self Report form, significant improvement in total QOL score was found and according to the post hoc analysis, a statistically significant increase was found between at baseline and one month later measurements $\left(t_{1}-t_{3} ; p=.008\right)$. In CG, a significant decrease was noted according to the PedsQL-Parent Report form and a significant reduction was found between $t_{1}$ and $t_{2}$ measurements according to the post hoc analyses $(p=.013)$. When compared with the groups, IG had significantly higher QOL level than CG according to the total scores of both the PedsQL-Child Self Report and Parent Report forms at the control measurements $(p=.045, p=.036$, respectively). In addition, IG had significantly higher QOL level especially in terms of nausea at discharge and one month later $(p<$ $.05)$. And, "communication" subtest of PedsQL improved in IG at the discharge when compared with baseline $(p<.05)$. Procedural and treatment anxiety level reduced in the both groups at the control measurements $(p<.05)$. Finally, "perceived physical appearance" subtest of PedsQL reduced in CG when compared with baseline $(p<.05)$ (Table 3).

The QOL level of the caregivers is shown in Table 4. There was a significant decrease in terms of total QOL level in CG $(p<.05)$, while significant improvements were found in IG in terms of physical abilities and emotional reactions $(p<.05)$. There was no statistically significant difference between two groups $(p>.05)$.

\section{Discussion}

In the present study, multimodal exercise program had positive effects on clinical status, depression, and QOL level of the children undergoing HSCT when compared with the 


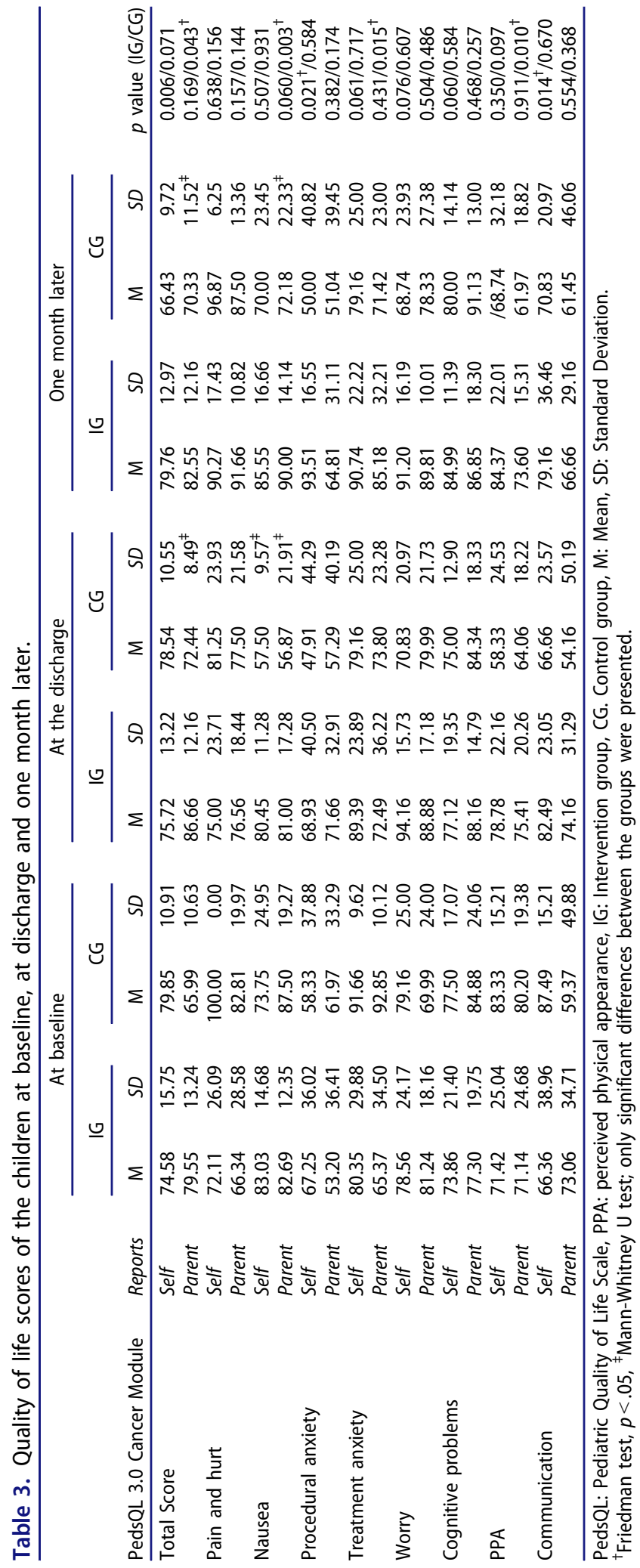


Table 4. Quality of life scores in the caregivers of the children at baseline and at discharge.

\begin{tabular}{|c|c|c|c|c|c|c|}
\hline \multirow[b]{2}{*}{ Nottingham Health Profile } & \multirow[b]{2}{*}{ Groups } & \multicolumn{2}{|c|}{ Baseline } & \multicolumn{2}{|c|}{ At the discharge } & \multirow[b]{2}{*}{$p$ value } \\
\hline & & M & $S D$ & M & $S D$ & \\
\hline \multirow[t]{2}{*}{ Total Score } & IG & 172.33 & 105.90 & 201.57 & 146.68 & 0.214 \\
\hline & CG & 170.73 & 116.76 & 225.32 & 106.44 & $0.017^{*}$ \\
\hline \multirow[t]{2}{*}{ Energy level } & IG & 44.57 & 44.91 & 40.35 & 42.67 & 0.581 \\
\hline & CG & 37.52 & 37.39 & 53.04 & 36.09 & 0.141 \\
\hline \multirow[t]{2}{*}{ Pain } & IG & 27.11 & 31.44 & 30.58 & 40.58 & 0.917 \\
\hline & CG & 16.04 & 30.23 & 20.06 & 30.64 & 0.144 \\
\hline \multirow[t]{2}{*}{ Physical abilities } & IG & 22.28 & 13.57 & 31.71 & 26.03 & $0.033^{*}$ \\
\hline & CG & 15.32 & 22.54 & 27.90 & 22.58 & 0.204 \\
\hline \multirow[t]{2}{*}{ Sleep } & IG & 35.50 & 29.82 & 39.92 & 21.92 & 0.465 \\
\hline & CG & 38.91 & 28.98 & 50.83 & 24.99 & 0.173 \\
\hline \multirow[t]{2}{*}{ Emotional reaction } & IG & 27.46 & 32.19 & 41.02 & 28.75 & $0.021^{*}$ \\
\hline & CG & 41.46 & 31.65 & 52.30 & 29.52 & 0.091 \\
\hline \multirow[t]{2}{*}{ Social isolation } & IG & 16.34 & 28.82 & 17.97 & 18.64 & 0.340 \\
\hline & CG & 21.46 & 27.31 & 21.17 & 24.90 & 0.414 \\
\hline
\end{tabular}

IG: Intervention group, CG: Control group, M: Mean, SD: Standard Deviation. Wilcoxon test. $*_{p}<.05$.

controls. This study also showed that the QOL level of the caregivers negatively affected during transplantation process. In addition, while supervised and multimodal exercise program was feasible and reliable, and participation rate to the exercise sessions was considerably high during inpatient period, home exercise program for children undergoing HSCT was not feasible after discharge from hospital.

Dimeo et al showed that aerobic exercise which performed on cycle ergometer during hospitalization had positive effects on adult patients' clinical status who underwent autologous HSCT. The duration of neutropenia, thrombocytopenia, and hospitalization; and severity of diarrhea and pain reduced in IG. It was also indicated that aerobic exercise can be safely performed daily for 30 minutes during hospitalization. ${ }^{25}$ In another study, which conducted on children undergoing HSCT, there was no statistically significant effect of exercise on duration of neutropenia and hospitalization, and necessity of thrombocyte or erythrocyte transfusion. ${ }^{13}$ In the present study, pain level was higher during hospitalization than at the all three time points: at baseline, at discharge, and one month later. Multimodal exercise program had positive effect on severity and duration of pain during hospitalization period when compared to CG in this study. We suggest that our exercise protocol, especially relaxation exercises during inpatient period, may help children to overcome complaint of pain. To our knowledge, relaxation exercises have not studied before in children undergoing HSCT. According to our results, these exercises are feasible and reliable for this population. On the other hand, in some cases especially in young children, relaxation exercises could not be effectively performed because of difficulty to understand. Not only relaxation training, but also other exercises including strengthening, endurance, and stretching were feasible for this population and multimodal exercise can be safely performed during hospitalization. In the literature, generally a single method was performed in exercise program. ${ }^{13,14} \mathrm{We}$ suggest that multimodal exercise program can be useful in clinical practice, due to the variety of symptoms of patients undergoing HSCT. Moreover, there was no side effect of the exercise program for children during exercise sessions. In the future studies, superiorities of these exercises over each other can be investigated and children were followed up for long period up to 100 days after HSCT. 
In studies conducted on adult HSCT patients, positive effects of exercise program were found on depression and anxiety level. ${ }^{26,27}$ This was also supported as a result of our work in the IG. These results may be attributed to increase in physical activity and can change emotional reactions and self-esteem. Moreover, relaxation exercises may contribute to the improvement of the emotional state of the children in the present study. There are rare data regarding the effects of exercise on fatigue level in children undergoing HSCT. Rosenhagen et al. found that exercise program performed totally $10.9 \pm 3.6$ sessions had led to decrease in children's fatigue level during inpatient period in children undergoing peripheral blood HSCT. In addition, exercise program was found feasible and reliable in children during hospitalization. ${ }^{14}$ In the present study, there was no significant effect of the exercise program on fatigue level in children. It was suggested that this may be associated with the difficulty in assessment of fatigue perception in children. Some problems in clinical practice with assessment of children's fatigue level were observed in this study; particularly, the assessment of fatigue level with visual expressions was not very understandable by the children. There is great need to develop methods for assessment of fatigue level in all age groups of Turkish children.

In a systematic review, it was indicated that children undergoing HSCT got tired more quickly during daily activities, and their QOL was affected negatively. They also emphasized that gaining exercise habit during treatment protocols positively affects QOL level of children with leukemia. ${ }^{28}$ Increase in QOL level was also reported as a result of exercise program consisted of aerobic exercise, running, walking, jumping, ball, and group games, which performed three times a week throughout 8 weeks in children who had undergone HSCT in the last 12 months. ${ }^{12}$

During assessing QOL level of children, it was proposed that both children and their parents should be asked in self-reported questionnaires. For this reason, the PedsQL 3.0 Cancer Module was selected in the present study and both children and their parents were questioned about children's QOL. According to the results, while HSCT process negatively affects QOL level of the children in CG, positive effects of exercise were shown on QOL level especially in terms of nausea, communication, and anxiety. When the groups were compared, the positive effect of exercise was shown only at the control measurements. Children have lots of side effects related to treatments during hospitalization period, especially infection, mucositis, and gastrointestinal complications. Because of these problems, positive effects of exercise on QOL may not be reached statistically significant level during inpatient period. After discharge, QOL level improved in IG and we suggest that positive effects of exercise continued up to one month later from discharge when compared with CG.

In a study, QOL level of caregivers decreased in terms of physical function, general health, vitality, and social functions before and after HSCT process. ${ }^{29}$ In the present study, the total QOL scores decreased in CG, while there was no difference in IG throughout HSCT process. We observed that caregivers of the children had a lot of responsibilities and also they worried about treatment and related complications and these problems may negatively affect their QOL. We suggest that positive effects of exercise program on children's problems may indirectly affect their caregiver and as a result, their perception of QOL was not deteriorated. In the future studies, the implementation of exercise or stress management trainings for caregivers should be emphasized. 
This study had some limitations. First of all, because of small number of children undergoing HSCT, the study sample was limited. In addition, because of some restrictions in HSCT service, exercises were performed in small hospital rooms. According to our observation and experience, this affected children's compliance to the exercise sessions and they got easily bored from the exercises. To increase participation to the exercise sessions, physiotherapist motivated children and their caregivers to continue exercise program. On the other hand, CG was not detailed followed up in terms of their physical activity level throughout HSCT and excluding social bias. In addition, compliance to the home exercise program was low in the present study. Because of fear of infection and isolation procedures after discharge, children could not often seen by the physiotherapist. In future studies, children may be followed more tightly to increase participation rate to exercises.

As a conclusion, when compared with controls, exercise program has positive effects on clinical status, depression, and QOL level of children undergoing HSCT. We suggest that multimodal supervised exercise program is an effective and useful method to increase feasibility. In addition, children should be tightly followed to increase participation to the home exercise programs. When considering caregivers, their QOL was negatively affected during hospitalization period, and we suggest that they need some interventions during HSCT process.

\section{Disclosure statement}

The authors report no conflict of interest.

\section{Acknowledgments}

The authors thank to all health professionals in the both hospitals which the present study was conducted.

\section{References}

1. Schmit Pokorny K. Expanding indications for stem cell transplantation. Semin Oncol Nurs. 2009;25:105-114. doi:10.1016/j.soncn.2009.03.002.

2. Copelan EA. Hematopoietic stem-cell transplantation. $N$ Engl J Med. 2006;354(17): 1813-1826. doi:10.1056/NEJMra052638.

3. Bevans MF, Marden S, Leidy NK, et al. Health-related quality of life in patients receiving reduced-intensity conditioning allogeneic hematopoietic stem cell transplantation. Bone Marrow Transplant. 2006;38(2):101-109. doi:10.1038/sj.bmt.1705406.

4. Heinonen H, Volin L, Zevon MA, et al. Stress among allogeneic bone marrow transplantation patients. Patient Educ Couns. 2005;56(1):62-71. doi:10.1016/j.pec.2003.12.007.

5. Hjermstad MJ, Knobel H, Brinch L, et al. A prospective study of health-related quality of life, fatigue, anxiety and depression 3-5 years after stem cell transplantation. Bone Marrow Transplant. 2004;34(3):257-266. doi:10.1038/sj.bmt.1704561.

6. Syrjala KL, Langer SL, Abrams JR, et al. Recovery and long-term function after hematopoietic cell transplantation for leukemia or lymphoma. JAMA. 2004;291(19):2335-2343. doi:10. 1001/jama.291.19.2335.

7. Kovalszki A, Schumaker GL, Klein A, et al. Reduced respiratory and skeletal muscle strength in survivors of sibling or unrelated donor hematopoietic stem cell transplantation. Bone Marrow Transplant. 2008;41(11):965-969. doi:10.1038/bmt.2008.15. 
8. Danaher EH, Ferrans C, Verlen E, et al. Fatigue and physical activity in patients undergoing hematopoietic stem cell transplant. Oncol Nurs Forum. 2006; 33:614-624. doi:10. 1188/06.ONF.614-624.

9. Broers S, Kaptein A, Le Cessie S, et al. Psychological functioning and quality of life following bone marrow transplantation: a 3-year follow-up study. J Psychosom Res. 2000;48(1): 11-21. doi:10.1016/S0022-3999(99)00059-8.

10. Ullrich CK, Rodday AM, Bingen KM, et al. Three sides to a story: child, parent, and nurse perspectives on the child's experience during hematopoietic stem cell transplantation. Cancer. 2017;123(16):3159-3166. doi:10.1002/cncr.30723.

11. Wiskemann J, Huber G. Physical exercise as adjuvant therapy for patients undergoing hematopoietic stem cell transplantation. Bone Marrow Transplant. 2008;41(4):321-329. doi:10. 1038/sj.bmt.1705917.

12. San Juan AF, Chamorro Vina C, Fernandez del Valle M, et al. Benefits of intrahospital exercise training after pediatric bone marrow transplantation. Int J Sports Med. 2008;29(5): 439-446. doi:10.1055/s-2007-965571.

13. Chamorro Vina C, Ruiz JR, Santana Sosa E, et al. Exercise during hematopoietic stem cell transplant hospitalization in children. Med Sci Sports Exerc. 2010;42(6):1045-1053. doi:10. 1249/MSS.0b013e3181c4dac1.

14. Rosenhagen A, Bernhörster M, Vogt L, et al. Implementation of structured physical activity in the pediatric stem cell transplantation. Klin Padiatr. 2011;223(03):147-151. doi:10.1055/ s-0031-1271782.

15. Yildiz Kabak V, Duger T, Uckan Cetinkaya D. Investigation of the effects of an exercise program on physical functions and activities of daily life in pediatric hematopoietic stem cell transplantation. Pediatr Blood Cancer. 2016;63(9):1643-1648. doi:10.1002/pbc.26038.

16. Rustler V, Hagerty M, Daeggelmann J, et al. Exercise interventions for patients with pediatric cancer during inpatient acute care: a systematic review of literature. Pediatr Blood Cancer. 2017;64(11):e26567. doi:10.1002/pbc.26567.

17. Kovacs M. The Children's Depression Inventory (CDI). Psychopharmacol Bull. 1985;21(4): 995-998.

18. Oy B. The Children's Depression Inventory: validity and reliability study. Turk J Psyc. 1991;2:132-136.

19. Wong DL, Baker CM. Pain in children: comparison of assessment. Pediatr Nurs. 1988; 14(1):9-17.

20. Varni JW, Burwinkle TM, Katz ER, et al. The PedsQL in pediatric cancer: reliability and validity of the pediatric quality of life inventory generic core scales, multidimensional fatigue scale, and cancer module. Cancer. 2002;94(7):2090-2106. doi:10.1002/cncr.10428.

21. Tanır MK, Kuğuoğlu S. Turkish validity and reliability of a pediatric quality of life cancer module for children ages 8-12 and parents. Asian Pacific J Cancer Prev. 2011;12:125-130.

22. Yıldız Kabak V, Yakut Y, Çetin M, et al. Reliability and validity of the Turkish version of PedsQL 3.0 Cancer Module for 2 to 7 year old and PedsQL 4.0 Generic Core Scale for 5 to 7 year old: the Hacettepe University experience. Turk J Haematol. 2016;33:228-235. doi:10. 4274/tjh.2015.0242.

23. Group E. European Group for Quality of Life Assessment and Health Measurement: European Guide to the Nottingham Health Profile. Brookwood, Surrey: Brookwood Medical Publications; 1993.

24. Kucukdeveci A, Mckenna SP, Kutlay S, et al. The development and psychometric assessment of the Turkish version of Nottingham Health Profile. Int J Rehabil Res. 2000;23: 31-38. doi:10.1097/00004356-200023010-00004.

25. Dimeo F, Fetscher S, Lange W, et al. Effects of aerobic exercise on the physical performance and incidence of treatment-related complications after high-dose chemotherapy. Blood. 1997;90(9):3390-3394.

26. Jarden M, Baadsgaard MT, Hovgaard DJ, et al. A randomized trial on the effect of a multimodal intervention on physical capacity, functional performance and quality of life in adult 
patients undergoing allogeneic SCT. Bone Marrow Transplant. 2009;43(9):725-737. doi:10. 1038/bmt.2009.27.

27. Dimeo FC, Stieglitz RD, Novelli-Fischer U, et al. Effects of physical activity on the fatigue and psychologic status of cancer patients during chemotherapy. Cancer. 1999;15:2273-2277. doi:10.1002/(SICI)1097-0142(19990515)85:10<2273::AID-CNCR24>3.0.CO;2-B.

28. van Brussel $M$, Takken $T$, Lucia $A$, et al. Is physical fitness decreased in survivors of childhood leukemia? A systematic review. Leukemia. 2005;19(1):13-17. doi:10.1038/sj.leu. 2403547.

29. El Jawahri AR, Traeger LN, Kuzmuk K, et al. Quality of life and mood of patients and family caregivers during hospitalization for hematopoietic stem cell transplantation. Cancer. 2015;121(6):951-959. doi:10.1002/cncr.29149. 